\title{
EphA3, induced by PC-1/PrLZ, contributes to the malignant progression of prostate cancer
}

\author{
RUIQIN WU ${ }^{1,2}$, HONGTAO WANG ${ }^{1,3}$, JIAN WANG $^{1}$, PENG WANG $^{1}$, FANG HUANG $^{1}$, \\ BANGXIANG XIE $^{1}$, YALI ZHAO ${ }^{1}$, SHANHU LI $^{1}$ and JIANGUANG ZHOU ${ }^{1}$ \\ ${ }^{1}$ Laboratory of Medical Molecular Biology, Beijing Institute of Biotechnology, Beijing 100850; \\ ${ }^{2}$ Evaluation and Research Center for Toxicology, Institute of Disease Prevention and Control, Academy of \\ Military Medical Science, Beijing 100071; ${ }^{3}$ State Key Laboratory of Experimental Hematology, \\ Institute of Hematology and Blood Diseases Hospital, Chinese Academy of Medical Sciences \\ and Peking Union Medical College, Tianjin 300020, P.R. China
}

Received June 27, 2014; Accepted August 28, 2014

DOI: 10.3892/or.2014.3482

\begin{abstract}
Our previous study revealed the potential linkage of $\mathrm{PC}-1 / \operatorname{PrLZ}$, a novel isolated prostate-specific gene, to the progression of prostate cancer $(\mathrm{PCa})$ in vitro and in vivo. To gain more insight into the mechanism of PC-1-induced promotion of $\mathrm{PCa}$, expression analysis of differential genes induced by PC-1 was scanned by microarray. Among all the differentially expressed genes, EphA3 was altered to the greatest extent. EphA3 has been identified to be associated with multiple tumor progression. However, little is known concerning the function of EphA3 in PCa. In the present study, we aimed to ascertain whether EphA3 is induced by PC-1 and the functional significance of EphA3 expression in PCa. We found that overexpression of PC-1 increased the amount of EphA3 and that knockdown of PC-1 led to a decrease in EphA3 in PCa cells. The functional significance and mechanisms by which EphA3 contributes to PCa was investigated in vitro using cell lines, and in vivo using a mouse model and clinical specimens. The results showed that EphA3 enhanced the proliferation and survival of LNCaP cells and suppression of EphA3 inhibited the survival of C4-2B cells. EphA3 enhanced the tumor development of LNCaP cells in null mice. A positive correlation between the levels of EphA3 and the Gleason grade of PCa was identified in clinical PCa specimens. In addition, cellular localization changed with Gleason grade. We further detected that EphA3 increased
\end{abstract}

Correspondence to: Professor Jianguang Zhou or Dr Shanhu Li, Laboratory of Medical Molecular Biology, Beijing Institute of Biotechnology, 27 Taiping Road, Haidian, Beijing 100850, P.R. China E-mail: zhou.jianguang@aliyun.com

E-mail: leeshanhu@hotmail.com

Key words: PC-1/PrLZ, EphA3, prostate cancer, Akt signaling pathway phosphorylation of Akt (Ser473 and Thr308), indicating that EphA3 activated the Akt pathway. Taken together, EphA3 was induced by PC-1 and contributed to the malignant progression of prostate cancer. Our results provide the first demonstration that EphA3 is a novel promoter of human prostate cancer development and progression.

\section{Introduction}

Prostate cancer (PCa) is currently the most commonly diagnosed cancer and the third leading cause of cancer-related deaths in males in developed countries, and the incidence is increasing in Asian countries $(1,2)$. PCa is a hormoneassociated disease that is dependent on signaling through androgen receptors (ARs) triggered by dihydrotestosterone (DHT). Our previous study focused on the role of PC-1, an androgen-induced prostate-specific gene. PC-1, also known as PrLZ (prostate leucine zipper gene), was first identified from 1,500 arrayed genes using cDNA differential expression microarray in LNCaP and C4-2 cells (3). PC-1 belongs to the tumor protein D52 (TPD52) family that has been reported to play important roles in exocytotic secretion and tumor progression (4). Different from the extensive expression of TPD52 in multiple tumor tissues and cell lines, PC-1 is predominantly expressed in the prostate, with only minimal expression in the gastrointestinal tract and other secretory glandular tissues (3). We previously revealed that $\mathrm{PC}-1$ caused malignant transformation of non-tumorigenic cells, promoted proliferation and survival in vitro and tumorigenicity in vivo, and contributed to androgen-independent progression and malignant phenotypes in prostate cancer cells, indicating its potential linkage to the progression of $\mathrm{PCa}$ (5-7). However, the detailed mechanism explaining how $\mathrm{PC}-1$ contributes to $\mathrm{PCa}$ progression remains unclear. To explore the molecular mechanisms of PC-1-induced promotion of $\mathrm{PCa}$, differentially expressed genes in PC-1overexpressing LNCaP cells were scanned by an Affymetrix microarray. Expression of EphA3, a member of the receptor tyrosine kinase (RTK) family, was markedly increased in the LNCaP-PC-1 cells. 
The EphA3 receptor has been reported to be overexpressed in malignant melanoma and lymphoid tumors (8), and to be mutated in tumors of the lung and breast (9). Soluble EphA3-Fc receptors and anti-EphA3 antibody can inhibit proliferation of tumor cells and decrease tumor volume in vivo $(10,11)$. In hematologic malignancies and glioblastoma, EphA3 is regarded as a promising therapeutic target (12-14). However, little is known concerning the function of EphA3 in PCa. Under normal conditions, higher amounts of EphA3 are produced in the bladder and prostate than in other human tissues (15). EphA3 expression was found to be increased by 39-fold in androgenindependent PCa cells (LNCaP-C81) compared with that in androgen-dependent PCa cells (LNCaP-C33) (16).

In the present study, we aimed to ascertain whether EphA3 is induced by the PCa-associated gene PC-1 and the functional significance of EphA3 expression in PCa.

\section{Materials and methods}

Plasmid construction and RNAi analysis. The coding region of the human EphA3 cDNA was cloned into the EcoRI and BamHI sites of pcDNA3.1(-)/Myc-His B vector (Invitrogen). The following double-stranded oligonucleotides were cloned into the pGP-U6-Hygro siRNA expression vector (Shanghai GenePharma Co., Ltd., Shanghai, China): EphA3, 5'-CACCGC GGTCAGCATCACAACTAATTTCAAGAGAATTAGTTGT GATGCTGACCGCTTTTTTG-3'. The negative control oligo, 5'-CACCGTTCTCCGAACGTGTCACGTTTCAAGAGAAC GTGACACGTTCGGAGAACTTTTTTG-3', had no significant homology to any human coding cDNA. All oligos were inserted into the pGP-U6-Hygro vector, and then transiently transfected into $293 \mathrm{~T}$ cells to examine the efficiency of RNAi by western blot analysis.

Cell culture, transfection and generation of stable cell lines. LNCaP and C4-2B cell lines were a generous gift from Leland Chung (Emory University, Atlanta, GA, USA). The LNCaP-PC-1 cell line was maintained in this laboratory, cultured in RPMI-1640 (Invitrogen) with $10 \%$ fetal bovine serum (FBS; HyClone). All cells were cultured at $37^{\circ} \mathrm{C}$, with $5 \% \mathrm{CO}_{2}$. The EphA3 cDNA vector was transfected into LNCaP cells using Lipofectamine 2000 (Invitrogen) according to the manufacturer's protocol. An empty vector was used as a control. Transfected LNCaP cells were selected in the presence of $1,000 \mu \mathrm{g} / \mathrm{ml} \mathrm{G} 418$ (Sigma). Stable clones were selected after 5 weeks, and clones overexpressing EphA3 were examined by western blot analysis.

Microarray hybridization and analysis. Total RNA from cultured LNCaP-PC-1 or control LNCaP-Neo cells was isolated individually using TRIzol reagent (Invitrogen, Carlsbad, CA, USA) and quantified spectrophotometrically at $260 / 280 \mathrm{~nm}$. The integrity of all RNA samples was evaluated on a $1 \%$ agarose gel, and all RNA samples were found to be pure with no degradation caused by the isolation procedure. Affymetrix GeneChips (Human Genome U133 Plus 2.0 arrays; Affymetrix, Santa Clara, CA, USA) were used for hybridization and data collection. The protocol was performed by the Microarray Facility at the Shanghai Biochip Co., Ltd., Shanghai, China.
Cell growth assays. To obtain growth curves, $2-3 \times 10^{3}$ cells/well were seeded in 96-well plates. Cell growth was measured using 3-(4,5-dimethylthiazol-2-yl)-2,5-diphenyltetrazolium bromide (MTT) assays according to the manufacturer's instructions (Amresco). Before testing, $20 \mu \mathrm{l}$ of MTT reagent $(2.5 \mathrm{mg} / \mathrm{ml}$ MTT in PBS; Amresco, Inc., Solon, OH, USA) was added, and the cells were incubated for a further $4 \mathrm{~h}$ at $37^{\circ} \mathrm{C}$. Then $150 \mu \mathrm{l}$ of dissolving reagent DMSO (Amresco) was added to dissolve the formazan crystals. The absorbance was detected at a wavelength of $490 \mathrm{~nm}$ on a microplate reader.

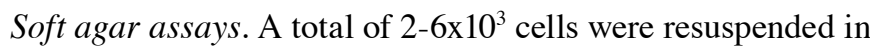
$3 \mathrm{ml}$ of $0.35 \%$ low melting point agarose (BD Biosciences) in RPMI-1640/10\% FBS and plated on top of a 2-ml underlayer of $0.5 \%$ agarose in the same medium in 6-well culture plates. After 3 weeks, the colonies containing $>50$ cells were counted.

Xenograft assays in nude mice. Male nude athymic BALB/c mice, 6-8 weeks old (Vital River Experimental Animals Technique Ltd., Beijing, China) were injected s.c. on the side of the abdomen with a total of $8 \times 10^{6}$ cells in an exponential growth phase suspended in $200 \mu 1$ serum-free RPMI-1640 containing Matrigel (1:1, v/v; BD Biosciences). Tumor volume measurements were recorded once a week and calculated as follows: L x W x H x 0.5236 .

Human prostate cancer tissue microarray and immunohistochemical staining. Two human prostate cancer tissue microarrays (Shanxi Chaoying Biotechnology Co., Ltd., Xi'an, China) were used to detected the expression of EphA3. A total of 110 formalin-fixed, paraffin-embedded benign prostate hyperplasia $(\mathrm{BPH})$ prostate cancer tissues and corresponding normal epithelia were included in the tissue microarray. Another microarray contained 207 dots of 69 clinical cases including normal prostate, $\mathrm{BPH}$, prostate cancer and adjacent normal prostate tissues in triplicate. Briefly, immunohistochemical (IHC) analysis was conducted as follows. Following deparaffinization, the samples were treated for antigen retrieval by the microwave method. A monoclonal antibody to EphA3 (Santa Cruz Biotechnology, Santa Cruz, CA, USA) (1:100) was used. Staining intensity and the percentage of immunoreactive cells were quantitated using image analysis ( $n=8$ images), and then the slides were sealed with Crystal/ Mount (Thermo Fisher Scientific, Pittsburgh, PA, USA) and analyzed separately by two pathologists.

Western blot analysis. Protein extracts were separated by SDS-PAGE and transferred to polyvinylidene difluoride membranes (Millipore, Bedford, MA, USA). Blots were blocked in the nitrocellulose membranes (Amersham Biosciences), then probed with antibodies against EphA3 (1:1000;), $\beta$-actin (1:2,000; Santa Cruz Biotechnology), phospho-Akt (pAkt; 1:1,000; Cell Signaling Technology), total Akt (1:1,000; Cell Signaling Technology) and tublin (1:1,000; Cell Signaling Technology). After a series of washes, blots were incubated with goat anti-mouse or anti-rabbit IgG antibody conjugated to horseradish peroxidase (Beijing Zhongshan Golden Bridge Biotechnology Co., Ltd., Beijing, China) then detected using an enhanced chemiluminescence kit (Pierce). 
Table I. Representative genes differentially expressed between PC-1-overexpressing LNCaP and control cells.

\begin{tabular}{lllr}
\multicolumn{1}{c}{ Description } & \multicolumn{1}{c}{$\begin{array}{c}\text { Representative } \\
\text { public ID }\end{array}$} & Fold-change \\
\hline Oncogene/tumor & suppressor genes & & \\
MAF & v-maf musculoaponeurotic fibrosarcoma oncogene homolog (avian) & AF055376 & 2.6 \\
NDRG1 & N-myc downstream regulated gene 1 & NM_006096 & 1.9 \\
RAP2B & RAP2B, member of the RAS oncogene family & AW005535 & 1.6 \\
ST7 & Suppression of tumorigenicity 7 & NM_018412 & -1.7 \\
Differentiation-associated genes & & \\
NKX3.1 & NK3 transcription factor related, locus 1 (Drosophila) & AF247704 & -1.4 \\
SPATS2 & Spermatogenesis associated, serine-rich 2 & NM_023071 & -1.4 \\
TEX27 & Testis expressed sequence 27 & NM_021943 & -1.4 \\
NCoA2 & Nuclear receptor coactivator 2 & NM_006540 & -1.7 \\
CALR & Calreticulin & NM_004343 & -1.6 \\
JAG1 & Jagged 1 (Alagille syndrome) & U77914 & -2.1 \\
NOTCH3 & Notch homolog 3 (Drosophila) & NM_000435 & -1.7 \\
Migration and & metastasis-associated genes & & \\
EphA3 & Eph receptor A3 & AF213459 & 42.2 \\
TUBA3 & Tubulin, $\alpha$ 3 & AF141347 & 1.7 \\
CDC42EP3 & CDC42 effector protein (Rho GTPase binding) 3 & AL136842 & 1.5 \\
ROCK1 & Rho-associated, coiled-coil containing protein kinase 1 & AV683882 & -1.4 \\
ITGA6 & Integrin, $\alpha$ 6 & AV733308 & -1.5 \\
Apoptosis-associated genes & & \\
FAF1 & Fas (TNFRSF6)-associated factor 1 & NM_007051 & -1.5 \\
PAK2 & p21 (CDKN1A)-activated kinase 2 & AI076186 & -1.5 \\
CASP9 & Caspase-9, apoptosis-related cysteine protease & AB015653 & -1.4 \\
BAG2 & BCL2-associated athanogene 2 & AF095192 & -1.5 \\
MDC1 & Mediator of DNA damage checkpoint 1 & AI673553 & -2.1 \\
DDA3 & Differential display and activated by p53 & BC001425 & -1.4 \\
TNFRSF21 & Tumor necrosis factor receptor superfamily, member 21 & BE568134 & -1.6 \\
\hline
\end{tabular}

Statistical analysis. Analyses were conducted using the statistical software SPSS13.0. All tests of significance were set at $\mathrm{P}<0.05$. Data analyses over time were undertaken by repeated measures analysis. For the staining of tissue microarrays, the average optical density, integral optical density, and area density data were subjected to ANOVA analysis, with the post hoc Scheffe test used for multiple comparisons between groups.

\section{Results}

EphA3 is induced by PC-1 in prostate cancer cells. Our previous results indicated the potential linkage of $\mathrm{PC}-1$ to the progression of $\mathrm{PCa}$ in vitro and in vivo. In order to gain more insight into the molecular mechanisms of PC-1-induced promotion of $\mathrm{PCa}$, we analyzed RNA from LNCaP-PC-1 and control LNCaP-Neo cells. Three cultures for each cell line were used for the RNA extraction. RNA was labeled and hybridized to Affymetrix U133 Plus 2.0 GeneChips. For each gene, the difference in expression level and the statistical significance of this difference were calculated. One hundrend and ninety-one genes were evaluated for 1.4 -fold relative increases and decreases in expression levels. These included 30 upregulated and 161 downregulated genes. Representative tumor-associated genes are shown in Table I. Expression levels of several oncogenes, such as RAP2B, MAF and NDRG (downstream of $\mathrm{N}-\mathrm{Myc}$ ), were increased by PC-1, while expression levels of several tumor suppressors including ST7 were decreased. Expression of apoptosis-associated genes, such as FAF1, CASP9, TNFRSF21 (DR6) was downregulated in the PC-1-overexpressing LNCaP cells. Expression levels of differentiation-associated genes, such as Nkx3.1, JAG1 and NOTCH3, were decreased by PC-1 as well. The data of the microarray were validated by RT-PCR and a portion of the results is shown in Fig. 1A. Notably, one of the migration and metastasis-associated genes, EphA3, was increased by 42-fold in the LNCaP-PC-1 cells.

Real-time PCR and western blot analysis were performed to confirm the PC-1-induced expression of EphA3. The results showed that EphA3 was induced by PC-1 in LNCaP cells at the mRNA (Fig. 1C) and protein (Fig. 1D) levels. We also detected the expression of PC-1 and EphA3 in six different prostate cell lines. As shown in Fig. 1B, no PC-1 or EphA3 expression was noted in benign prostatic hyperplasia (BPH1) cells. 
A

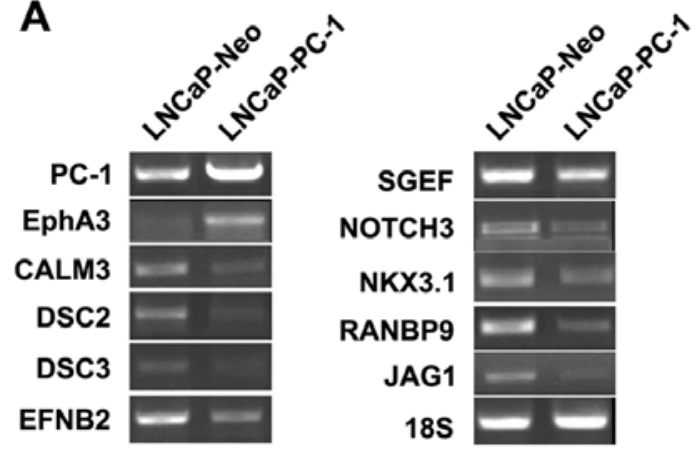

C

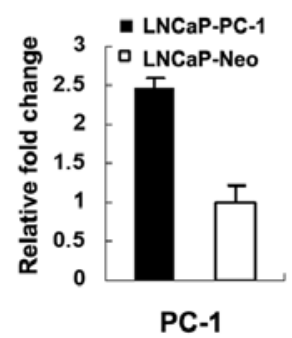

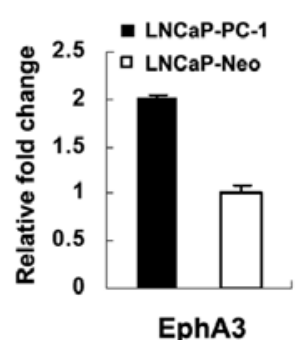

B

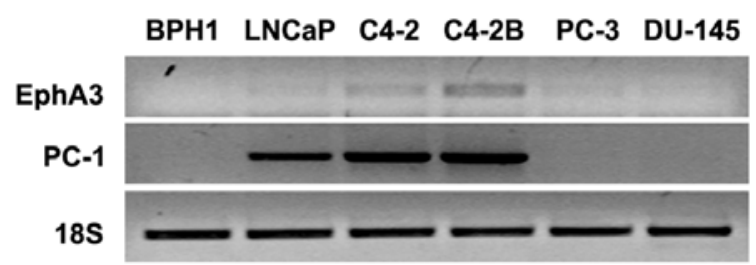

D

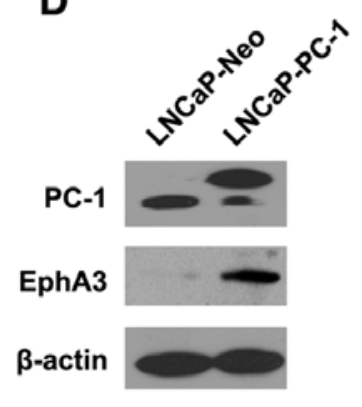

E

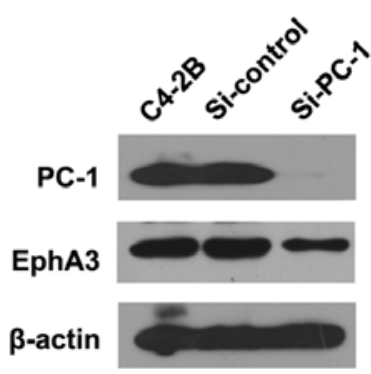

Figure 1. Expression of EphA3 is induced by PC-1 in prostate cancer cells. (A) RT-PCR results of the expression levels of the genes of interest selected from the microarray which scanned the differentially expressed genes between PC-1-overexpressing LNCaP cells and control cells. (B) Analysis of the mRNA levels of EphA3 and PC-1 in benign prostatic hyperplasia (BPH1) cells and LNCaP, C4-2, C4-2B, PC-3 and DU-145 prostate cancer cell lines. (C) Real-time PCR result of EphA3 and PC-1 expression in the LNCaP-PC-1 and LNCaP-Neo cells. (D) Western blot analysis of EphA3 and PC-1 expression in the LNCaP-PC-1 and LNCaP-Neo cells. (E) Western blot analysis of EphA3 and PC-1 expression in the C4-2B cells transfected with PC-1 siRNA or the negative control or in the parental $\mathrm{C} 4-2 \mathrm{~B}$ cells.

The LNCaP cell line, established from a metastatic deposit in a lymph node, is androgen-dependent, non-metastatic, and weakly tumorigenic (17). The LNCaP lineage-derived sublines C4-2 and C4-2B, obtained from tumor-stoma interaction, possess the capabilities of androgen-independent growth and distant organ metastasis $(18,19)$. The mRNA expression of PC-1 increased progressively in the LNCaP, C4-2 and C4-2B cells. PC-3 and DU145 are two prostate cancer cell lines derived from lumbar and central nervous system metastasis, respectively, and are both androgen-independent. There was minimal expression of EphA3 and undetectable PC-1 expression in these two cell lines. Consistent with the mRNA level, the EphA3 protein was increased in the $\mathrm{PC}$-1-overexpressing LNCaP cells compared with the control. PC-1-targeted siRNA was used to suppress the PC-1 expression in C4-2B cells. The amount of EphA3 protein was decreased in the si-PC1-transfected C4-2B cells compared with the negative RNAi control and parental C4-2B cells. In another part of our study, a 2011-bp length of the EphA3 promoter sequence was cloned into a luciferase report gene vector, and PC-1 was found to enhance the activity of the EphA3 promoter in luciferase assays, suggesting that $\mathrm{PC}-1$ may induce the EphA3 expression through increasing the promoter activity (20). Taken together, these results above indicate that EphA3 is induced by PC- 1 in prostate cancer cells.

Overexpression of EphA3 enhances the proliferation and survival of $L N C a P$ cells. In view of the fact that PC-1 promoted prostate cancer progression and PC-1-induced EphA3 expres- sion, we were interested in investigating the role of EphA3 in prostate cancer. For this purpose, EphA3 stably expressing and control LNCaP cell lines were constructed by transfection of an EphA3-expressing vector or a mock vector. Two LNCaPEphA3 clones (\#23 and \#25) were determined to have higher levels of EphA3 expression than the control cells (LNCaP-Neo) (Fig. 2A). MTT assays were performed to evaluate the growth of the transfected cells. The growth rate of the LNCaP-EphA3 cells increased by 1.6-, 2.0- and 2.2-fold compared with the control at 2, 4 and 6 days, respectively (Fig. 2B). In the plate colony-formation assay, it was found that EphA3 markedly enhanced the colony-formation ability of the LNCaP cells. LNCaP-EphA3 cells formed more and larger colonies than the control after 10 days in culture (Fig. 2C and D). Based on these results, the effect of EphA3 expression on the anchorageindependent growth of $\mathrm{LNCaP}$ cells was evaluated in colony formation assays. As shown in Fig. 2E, LNCaP-EphA3 cells exhibited enhanced clonogenicity in semi-solid agar compared with the mock-transfected control cells. These results suggest that overexpression of EphA3 enhances the proliferation and survival of LNCaP cells.

Knockdown of EphA3 suppresses the proliferation and survival of C4-2B cells. Due to its high expression of EphA3 protein in C4-2B cells, they were chosen to construct the EphA3 knockdown cell model. EphA3 targeted siRNA was transfected into C4-2B cells, and the expression of EphA3 was markedly suppressed compared with the si-control and parental cells (Fig. 3A). In the MTT assay, the growth rate of the si-EphA3- 
A
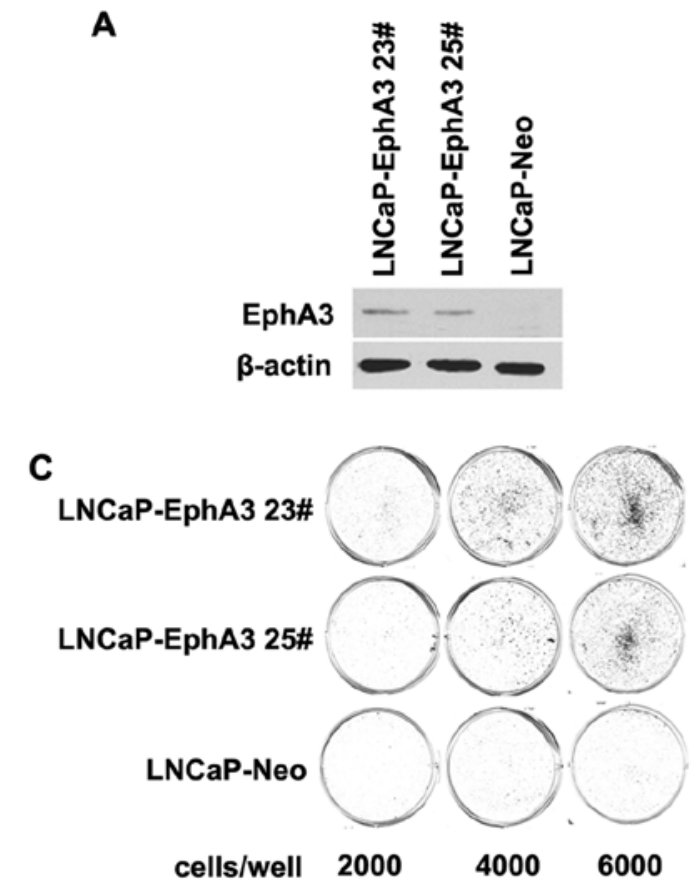

B

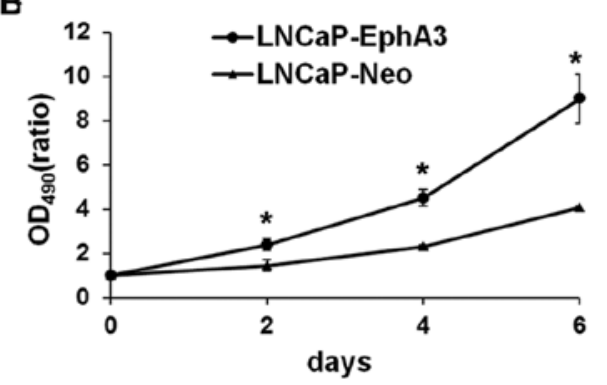

D

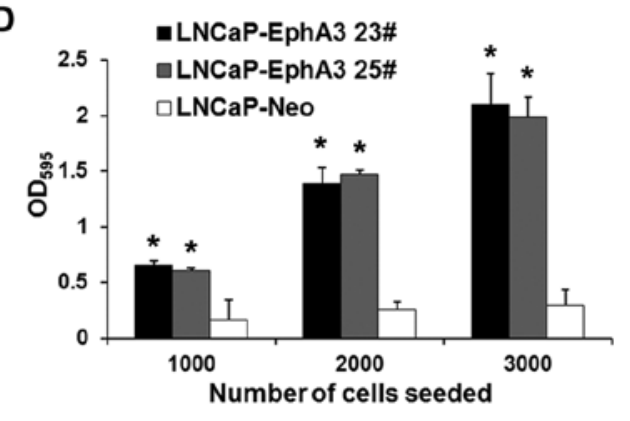

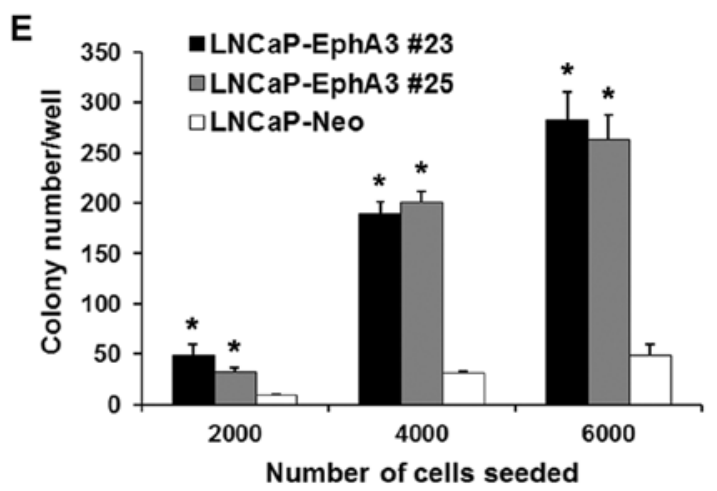

Figure 2. Overexpression of EphA3 enhances the proliferation and survival of LNCaP cells. (A) LNCaP cells were transfected with an EphA3-expressing vector or a control vector, and then were selected with G418. Western blot analysis was performed to analyze expression of EphA3 vs. $\beta$-actin in two selected clones of LNCaP-EphA3 and LNCaP-Neo stable cell lines. (B) Cell growth curves calculated from MTT assay data for LNCaP cells overexpressing EphA3 and control. (C and D) Plate colony-formation assays. Cells were seeded onto plates, and after 14 days of growth, the cells were fixed and stained using crystal violet. (E) Soft agar assays. The colony-forming capability was determined as detailed in Materials and methods. Data in D and E are presented as means \pm SD. ${ }^{*} \mathrm{P}<0.05$, compared with the control cells.

transfected cells was decreased compared with this rate in the si-control cells (Fig. 3B). In the plate colony-formation assay, EphA3 suppression led to a markedly reduction in colonyformation ability of the C4-2B cells (Fig. 3C and D). Similarly, EphA3 suppression decreased the clonogenicity of the C4-2B cells compared with that noted in the control cells (Fig. 3E). These results suggest that knockdown of EphA3 suppresses the proliferation and survival of $\mathrm{C} 4-2 \mathrm{~B}$ cells, coinciding with the results in the $\mathrm{LNCaP}$ cells.

EphA3 enhances the tumorigenicity of prostate cancer cells in vivo. EphA3 enhances the proliferation and survival of prostate cancer cell in vitro. Furthermore, we determined the in vivo tumor growth and tumor take of EphA3-overexpressing LNCaP cells in nude mice. Similar to PC-1, data from the s.c. model indicated that ectopic EphA3 expression facilitated the tumor take rate and accelerated tumor growth in the LNCaPEphA3 cells (Fig. 4A). Detectable tumors were observed within 5 weeks after injection (Fig. 4B). Visible tumors were noted in 4/7 mice by the end of 9 weeks. In contrast, the control LNCaP cells did not form tumors. In addition, tumor-bearing mice also exhibited a more emaciated phenotype than mice without tumors. These data indicate that EphA3 enhances the tumorigenicity of prostate cancer cells in vivo.

EphA3 is overexpressed in clinical prostate cancer specimens and its expression and cellular localization are correlated with the Gleason grade of prostate cancer. A tissue array containing 110 clinical specimens of BPH, PCa and adjacent normal prostate tissues, was analyzed by immunohistochemistry. Excluding 4 cases of non-adenocarcinoma, medium to strong expression of EphA3 was observed in 59/73 (80.8\%) cancer tissues, while weak expression of EphA3 was detected in 8/27 (29.6\%) normal prostate tissues (Table II). Immunohistochemisty staining was quantitated using image analysis software, and statistical analyses identified a positive 
A

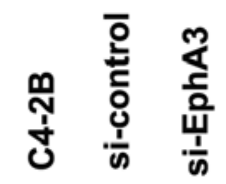

EphA3

$\beta$-actin

C

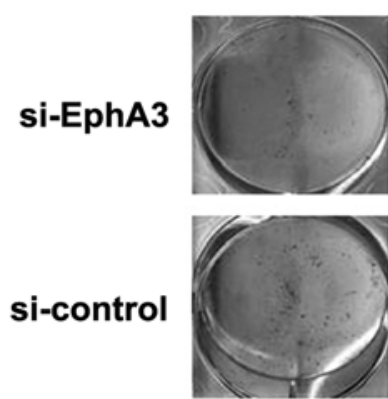

cells/well

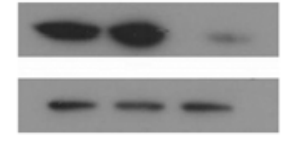

2000
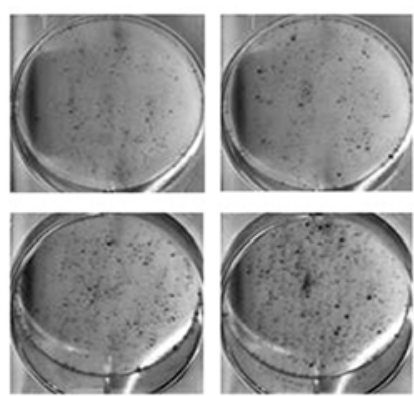

4000

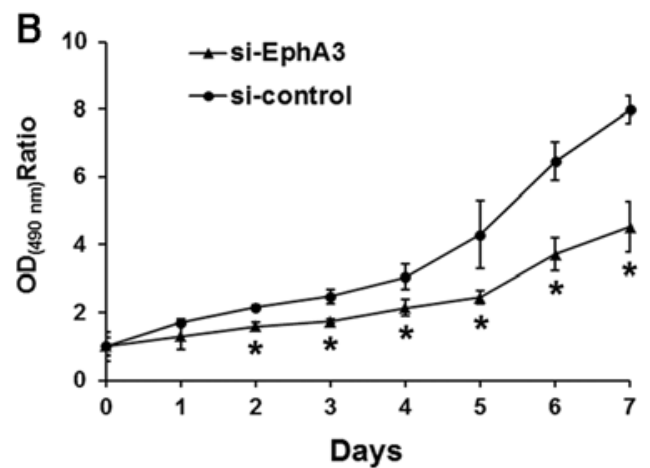

D

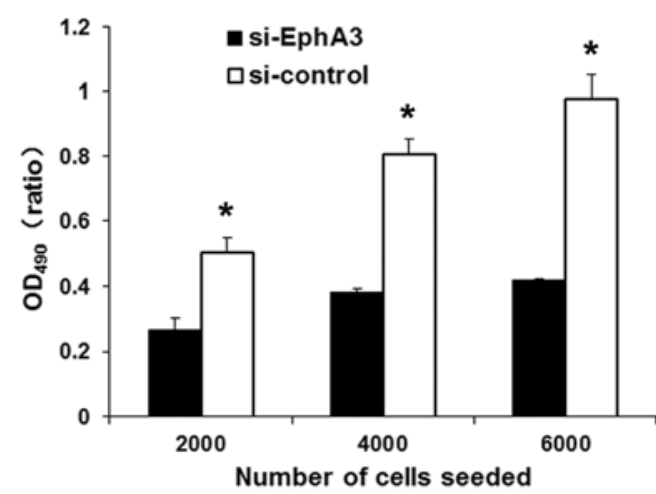

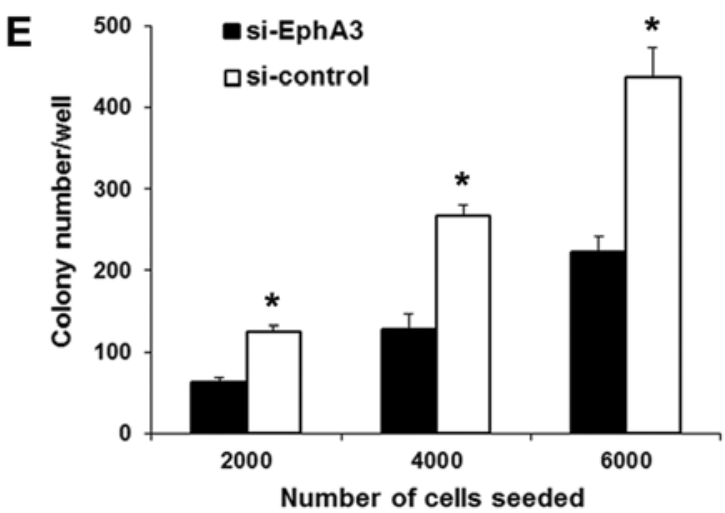

Figure 3. Knockdown of EphA3 suppresses the proliferation and the survival of C4-2B cells. (A) C4-2B cells were transfected with the si-EphA3-expressing vector (si-EphA3) or control vector (si-control). Western blot analysis was performed to analyze the expression of EphA3 vs. $\beta$-actin in the si-EphA3/ si-control transfected and parental C4-2B cells. (B) C4-2B cells were transfected with si-EphA3 or si-control, and the cells were seeded onto 96-well plates after $24 \mathrm{~h}$. Cells were counted by MTT assay daily. (C and D) Plate colony-formation assays. One day after transfection, the cells were seeded on 6-well plate, and after 10 days of growth, the cells were fixed and stained using crystal violet. (E) Soft agar assays. One day after transfection, the cells were seeded on 6 -well plate, and after 14 days of growth, the colonies containing $>50$ cells were counted. Data in $\mathrm{D}$ and $\mathrm{E}$ are presented as means $\pm \mathrm{SD}$. $\mathrm{P}<0.05$, compared with the control cells.

Table II. Expression of EphA3 in human prostate specimens.

\section{Expression of EphA3}

Cases, $\mathrm{n} \quad$ Positive, $\mathrm{n}(\%) \quad$ Negative, $\mathrm{n}(\%)$

\begin{tabular}{lrcr}
\hline Normal & 27 & $8(29.6)$ & $19(70.4)$ \\
BPH & 6 & $1(16.7)$ & $5(83.3)$ \\
Cancer & 73 & $59(80.8)^{\mathrm{a}}$ & $14(19.2)$ \\
\hline
\end{tabular}

${ }^{\mathrm{a}} \mathrm{P}<0.01$, compared with normal tissues and benign prostate hyperplasia (BPH). correlation between levels of EphA3 and the Gleason grade of PCa (Fig. 5A). Cellular localization of EphA3 was also found to change as the grade of PCa increased. EphA3 was expressed preferentially in the cytoplasm in Gleason 1 samples, EphA3 was distributed equally between the cytoplasm and nucleus in Gleason 2 and 3 samples, predominantly nuclear staining was observed in Gleason 4 samples, and a nodular expression pattern was observed in a cluster of cell nuclei in Gleason 5 samples (Fig. 5B). To confirm these results, a second tissue array containing 207 dots of 69 clinical specimens (triplicate for each), including normal prostate, BPH, $\mathrm{PCa}$, and adjacent normal prostate tissues was assembled and analyzed. Results 
A

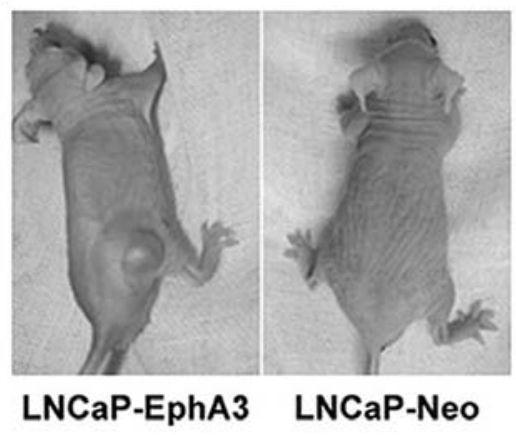

B

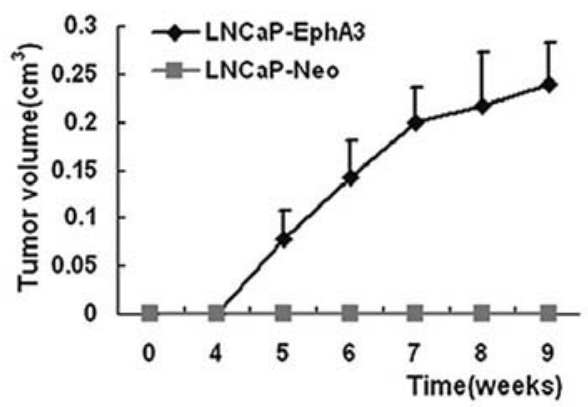

Figure 4. EphA3 enhances the tumorigenicity of prostate cancer cells in vivo. LNCaP-EphA3 cells were injected s.c. into the right flank of male nude mice, or LNCaP-Neo cells were injected as the control. Tumor volume was measured weekly. (A) Representative images of nude mice incubated for 8 weeks with subcutaneously implanted LNCaP-EphA3 cells and control cells. (B) Tumor growth curves for LNCaP-EphA3 mice ( $\mathrm{n}=7$ ) and control mice ( $\mathrm{n}=7)$ monitored weekly.

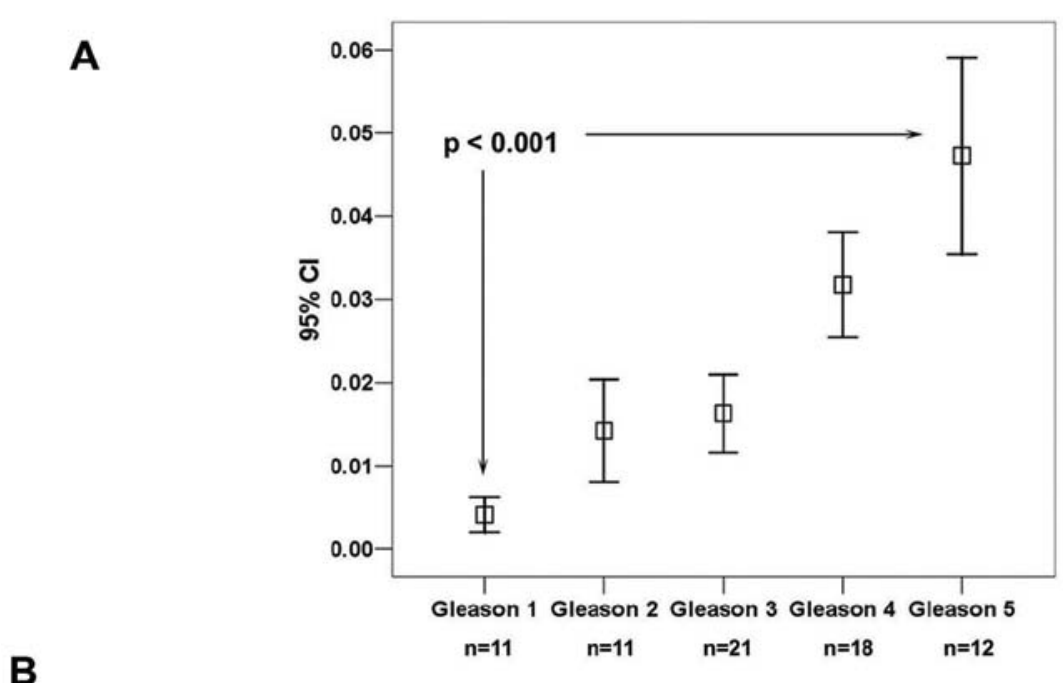

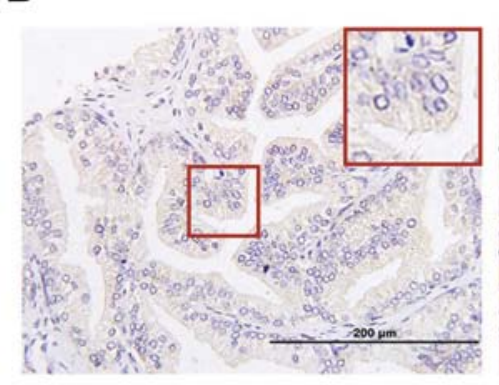

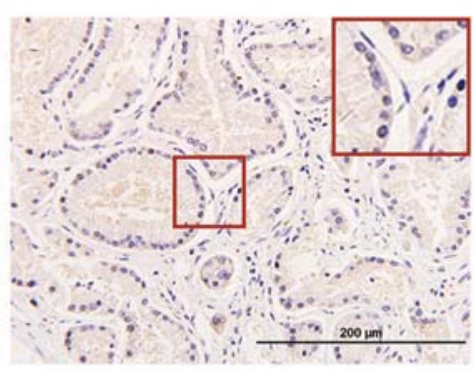

Adenocarcinoma, gleason1

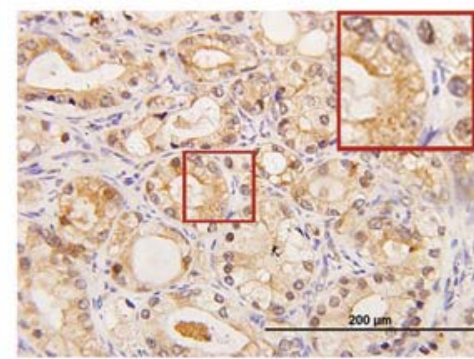

Adenocarcinoma, gleason 3

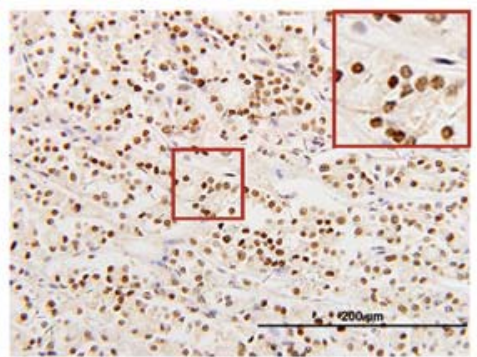

Adenocarcinoma, gleason4

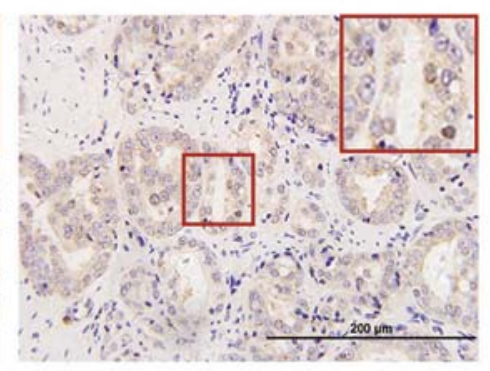

Adenocarcinoma, gleason2

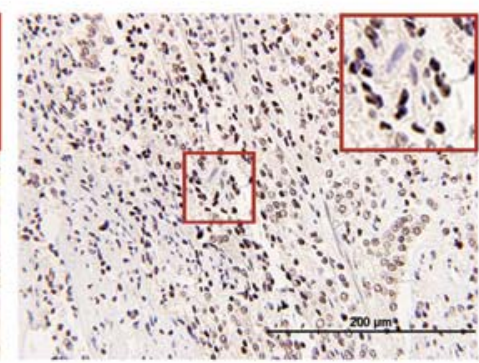

Adenocarcinoma, gleason5

Figure 5. Expression of EphA3 in the clinical PCa specimens. A tissue array containing 110 clinical specimens of BPH, PCa and adjacent normal prostate tissues was analyzed by immunohistochemistry. (A) Values of the $95 \%$ confidence interval (CI) associated with various Gleason grades of PCa tissues. A positive correlation between expression of EphA3 and Gleason grade was observed ( $\mathrm{P}<0.001)$. (B) Representative photomicrographs of EphA3 immunoreactivity in adenocarcinoma tissues of various Gleason grades compared to normal prostate tissue (original magnification, x200). Scale bar, $200 \mu \mathrm{m}$. 
B

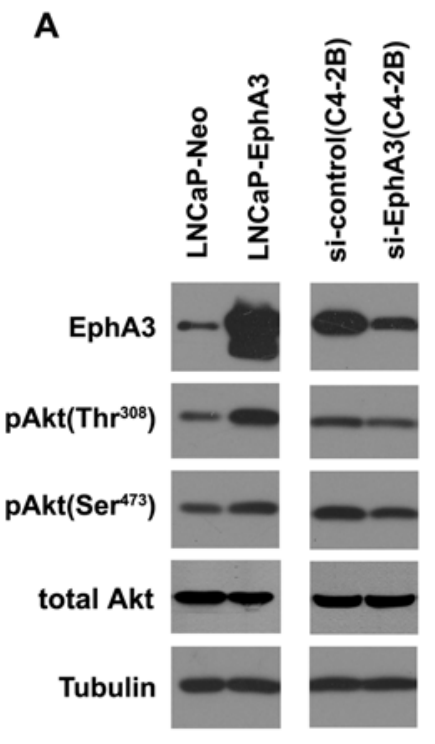

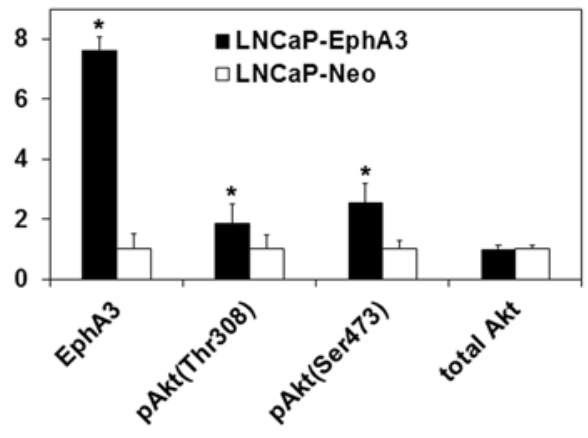

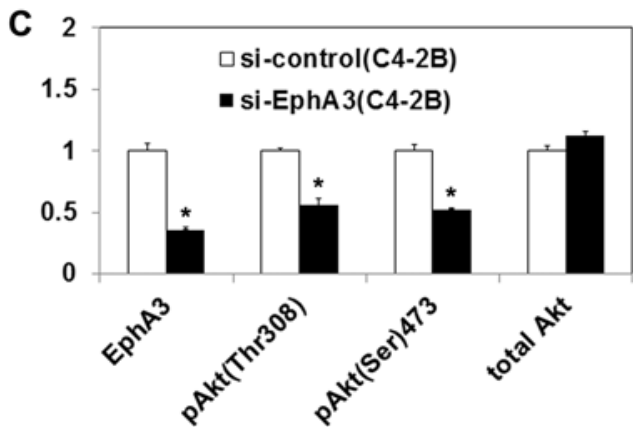

Figure 6. Effect of EphA3 on the Akt signaling pathway. (A) Expression levels of EphA3, phosphorylated Akt (pAkt) and total Akt were detected by western blot analysis in LNCaP-EphA3 and LNCaP-Neo stable cell lines and C4-2B cells transfected with si-EphA3 or si-control. Tubulin was used as a loading control. (B and C) Quantitation of western blot results by Quantity One software. "P<0.05, compared with the control.

consistent with the first array were observed (data not shown). EphA3 was overexpressed in PCa specimens and its expression increased and its cellular localization changed as the Gleason grade was elevated.

EphA3 activates the Akt signaling pathway. The Akt signaling pathway has been well-characterized for its role in promoting cell growth, invasion and metastasis during tumorigenesis (21-23). We examined the impact of EphA3 on the Akt pathway. Overexpression of EphA3 was found to increase the phosphorylation of Akt at Ser473 and Thr308 in LNCaP cells. The phosphorylation of Akt was deduced with inhibition of EphA3 in the C4-2B cells (Fig. 6A). The quantitation of the expression of the genes showed that the expression levels of EphA3, pAkt (Ser473), pAkt (Thr308) changed significantly (Fig. 6B and C). These results indicate that EphA3 activates the Akt signaling pathway.

\section{Discussion}

EphA3 is a member of the Eph receptor subfamily, the largest family of vertebrate receptor protein tyrosine kinases. Increasing evidence has demonstrated a strong correlation between dysregulation of Eph family members and tumorigenesis and progression. EphA3 was originally isolated from the membrane of pre-B cell acute lymphoblastic leukemia cells and was associated with axon-guiding events (24). More recently, EphA3 has been found to be related with multiple tumors, such as malignant melanoma, lymphoid tumors, lung and breast cancer. Although several studies have reported that EphA3 is expressed at higher levels in PCa cells, there is limited data regarding the functional significance of EphA3 expression. Even in other types of tumors, the role of EphA3 has not been investigated as extensively as other members of the Eph receptor family. The present study represents the first investigation of EphA3 function in the development and progression of $\mathrm{PCa}$.

In the present study, EphA3 was identified in a comparative gene array analysis of PC-1-overexpressing LNCaP and control cells. PC-1 is a novel isolated prostate-specific gene (17). Contrary to its low expression in the androgendependent human prostate cancer cell line LNCaP, the level of PC-1 is differentially elevated in the lineage-related but androgen-independent C4-2 human prostate cancer cells, which are capable of forming tumors in castrated mice (25). PC-1 expression enhanced the proliferation and invasion capability in vitro and also increased the tumorigenicity in an in situ prostate cancer animal model, which indicated that PC-1 expression contributes to the malignancy progression of prostate cancer (26).

In the present study, EphA3 was not detected in cells derived from $\mathrm{BPH}$, yet was expressed at high levels in $\mathrm{PCa}$ cell lines, LNCaP and C4-2B. EphA3 expression was also detected in PCa patient tissues, and not in adjacent normal tissues or BPH tissue specimens. A positive correlation between the extent of EphA3 expression and the Gleason grade of PCa was also observed, which is consistent with data from melanoma cell lines derived from distant metastases that have been shown to express higher levels of EphA3 compared to primary melanomas (27). In vitro and in vivo 
assays also demonstrated that overexpression of EphA3 enhanced the survival and tumorigenicity of PCa cells. Based on these data, as well as evidence that EphA3 can induce the malignant transformation of NIH3T3 cells (28), we conclude that EphA3 is a potential oncogene that has an important role in the development and malignant progression of PCa. The Akt signaling pathway plays an important role in promoting cell growth, invasion and metastasis during tumorigenesis (21-23). In the present study, EphA3 increased phosphorylation of Akt in LNCaP and C4-2B cells, suggesting the association of EphA3-induced progression of PCa with activation of the Akt pathway.

In conclusion, we demonstrate that EphA3 is induced by PC- 1 and is able to promote the development and progression of PCa. These findings offer the the potential to identify novel therapeutic targets of EphA3-positive prostate cancers to improve patient treatment and survival.

\section{Acknowledgements}

The present study was supported in part by the National Science and Technology Major Project of the Ministry of Science and Technology of China (2012ZX10003008002 to S.H.L.) and the National Natural Science Foundation of China (81372770 to J.G.Z. and 81371740 to J.W.).

\section{References}

1. Jemal A, Bray F, Center MM, Ferlay J, Ward E and Forman D Global cancer statistics. CA Cancer J Clin 61: 69-90, 2011.

2. Baade PD, Youlden DR, Cramb SM, Dunn J and Gardiner RA: Epidemiology of prostate cancer in the Asia-Pacific region. Prostate Int 1: 47-58, 2013.

3. Wang R, Xu J, Saramaki O, et al: PrLZ, a novel prostate-specific and androgen-responsive gene of the TPD52 family, amplified in chromosome 8q21.1 and overexpressed in human prostate cancer. Cancer Res 64: 1589-1594, 2004.

4. Li L, Xie H, Liang L, et al: Increased PrLZ-mediated androgen receptor transactivation promotes prostate cancer growth at castration-resistant stage. Carcinogenesis 34: 257-267, 2013.

5. Zhang H, Wang J, Pang B, et al: PC-1/PrLZ contributes to malignant progression in prostate cancer. Cancer Res 67: 8906-8913, 2007.

6. Wang J, Zhang H, Liang RX, et al: Identification and characterization of the novel human prostate cancer-specific PC-1 gene promoter. Biochem Biophys Res Commun 357: 8-13, 2007.

7. Yu L, Shi QG, Qian XL, et al: PC-1 enhances c-myc gene expression in prostate cancer cells. Yi Chuan 32: 348-352, 2010 (In Chinese).

8. Wimmer-Kleikamp SH and Lackmann M: Eph-modulated cell morphology, adhesion and motility in carcinogenesis. IUBMB Life 57: 421-431, 2005.

9. Wood LD, Calhoun ES, Silliman N, et al: Somatic mutations of GUCY2F, EPHA3, and NTRK3 in human cancers. Hum Mutat 27: 1060-1061, 2006.
10. Brantley DM, Cheng N, Thompson EJ, et al: Soluble Eph A receptors inhibit tumor angiogenesis and progression in vivo. Oncogene 21: 7011-7026, 2002.

11. Vearing C, Lee FT, Wimmer-Kleikamp S, et al: Concurrent binding of anti-EphA3 antibody and ephrin-A5 amplifies EphA3 signaling and downstream responses: potential as EphA3specific tumor-targeting reagents. Cancer Res 65: 6745-6754, 2005.

12. Keane N, Freeman C, Swords R and Giles FJ: EPHA3 as a novel therapeutic target in the hematological malignancies. Expert Rev Hematol 5: 325-340, 2012.

13. Guan M, Liu L, Zhao X, et al: Copy number variations of EphA3 are associated with multiple types of hematologic malignancies. Clin Lymphoma Myeloma Leuk 11: 50-53, 2011.

14. Day BW, Stringer BW, Al-Ejeh F, et al: EphA3 maintains tumorigenicity and is a therapeutic target in glioblastoma multiforme. Cancer Cell 23: 238-248, 2013.

15. Hafner C, Schmitz G, Meyer S, et al: Differential gene expression of Eph receptors and ephrins in benign human tissues and cancers. Clin Chem 50: 490-499, 2004.

16. Singh AP, Bafna S, Chaudhary K, et al: Genome-wide expression profiling reveals transcriptomic variation and perturbed gene networks in androgen-dependent and androgen-independent prostate cancer cells. Cancer Lett 259: 28-38, 2008.

17. Zhang D, He D, Xue Y, et al: PrLZ protects prostate cancer cells from apoptosis induced by androgen deprivation via the activation of Stat3/Bcl-2 pathway. Cancer Res 71: 2193-2202, 2011.

18. van Bokhoven A, Varella-Garcia M, Korch C, et al: Molecular characterization of human prostate carcinoma cell lines. Prostate 57: 205-225, 2003

19. Wu X, Gong S, Roy-Burman P, Lee P and Culig Z: Current mouse and cell models in prostate cancer research. Endocr Relat Cancer 20: R155-R170, 2013.

20. Wu RQ, Li QM, Shi QG, Zhang H, Qian XL, Yu L and Zhou JG: PC-1 can enhance transcription of EphA3, a member of RTK family, in prostate cancer cells. Chin J Biochem Mol Biol 25: 633-639, 2009.

21. Jeong SJ, Pise-Masison CA, Radonovich MF, Park HU and Brady JN: Activated AKT regulates NF-kappaB activation, p53 inhibition and cell survival in HTLV-1-transformed cells. Oncogene 24: 6719-6728, 2005.

22. Viglietto G, Motti ML, Bruni P, et al: Cytoplasmic relocalization and inhibition of the cyclin-dependent kinase inhibitor $\mathrm{p} 27^{\mathrm{Kipl}}$ by $\mathrm{PKB} / \mathrm{Akt}$-mediated phosphorylation in breast cancer. Nat Med 8: 1136-1144, 2002.

23. Vivanco I and Sawyers CL: The phosphatidylinositol 3-kinase AKT pathway in human cancer. Nat Rev Cancer 2: 489-501, 2002.

24. Wicks IP, Wilkinson D, Salvaris E and Boyd AW: Molecular cloning of HEK, the gene encoding a receptor tyrosine kinase expressed by human lymphoid tumor cell lines. Proc Natl Acad Sci USA 89: 1611-1615, 1992.

25. Wang R, Xu J, Mabjeesh N, et al: PrLZ is expressed in normal prostate development and in human prostate cancer progression. Clin Cancer Res 13: 6040-6048, 2007.

26. Li L, Zhang D, Zhang L, et al: PrLZ expression is associated with the progression of prostate cancer LNCaP cells. Mol Carcinog 48: 432-440, 2009

27. Easty DJ and Bennett DC: Protein tyrosine kinases in malignant melanoma. Melanoma Res 10: 401-411, 2000.

28. Li QM, Wu RQ, Pan DR and Zhou JG: Construction and analysis of EphA3 gene stable-expressed NIH3T3 cell model. Lett Biotech 20: 609-611, 2009. 